\title{
Automatic Classification of Red Blood Cells using Gaussian Mixture Densities
}

\author{
J. Dahmen ${ }^{1}$, J. Hektor ${ }^{2}$, R. Perrey ${ }^{1}$, H. Ney ${ }^{1}$ \\ ${ }^{1}$ Lehrstuhl für Informatik VI, RWTH Aachen - University of Technology, Germany \\ ${ }^{2}$ Department of Physiology, RWTH Aachen - University of Technology, Germany \\ Email: dahmen@informatik.rwth-aachen.de
}

\begin{abstract}
In this paper we present an invariant statistical approach to classifying red blood cells (RBC). Given a database of 5062 grayscale images, we model the distribution of the observations by using Gaussian mixture densities within a Bayesian framework. As invariance is of great importance when classifying RBC, we use a Fourier-Mellin based approach to extract features which are invariant with respect to $2 \mathrm{D}$ rotation, shift and scale. To prove the efficiency of our approach, we also apply it to the widely used US Postal Service handwritten digits recognition task, obtaining state-of-the-art results.
\end{abstract}

Keywords: statistical pattern recognition, invariant object recognition

\section{Introduction}

In standard tests, drugs that induce shape changes to RBC are often used to examine whether the cell membrane still acts in a well known way. This is done by comparing induced shape changes with the known behaviour on drugs [1]. This comparison is usually performed by a human expert and therefore time and cost consuming, stressing the need for automatic classification. In this paper, we propose an invariant statistical approach to automatic RBC classification. Given a set of 5062 images (which were labelled as stomatocyte, echinocyte or discocyte by an expert), we model the distribution of the observed training data using Gaussian mixture densities (GMD), where classification is achieved by embedding the model into a Bayesian framework. As invariance plays an important role in successfully classifying RBC (because position and orientation may vary during observation), we extract Fourier-Mellin based features from the images, thus being invariant with respect to $2 \mathrm{D}$ rotation, scale and translation (RST). Using the proposed classifier, we obtain an error rate of $13.6 \%$ on the RBC data. This seemingly high error rate is still considerably lower than the human error rate of $>20 \%$ [2]. To prove the efficiency of our approach, we also apply it to the widely used US Postal Service handwritten digits recognition task (USPS). The obtained error rate of $3.4 \%$ is one of the best USPS results published so far (cp. Chapter 5).

\section{Databases used in our Experiments}

For our experiments, we use a database of $5062 \mathrm{RBC}$ that were expert labelled as stomatocyte, echinocyte or discocyte, where each cell is represented by a $128 \times 128$ pixels sized grayscale image (see Fig. 1). The images were taken in a capillary 
Table1. Some Properties of the Fourier Transformation

\begin{tabular}{|c|c|}
\hline Signal & Fourier Transform \\
\hline \hline$f\left[x-x_{0}, y-y_{0}\right]$ & $\mathcal{F}(u, v) \cdot \exp \left(-2 \pi i\left(x_{0} u+y_{0} v\right)\right)$ \\
\hline$f[\beta x, \beta y]$ & $\frac{1}{\beta^{2}} \mathcal{F}\left(\frac{u}{\beta}, \frac{v}{\beta}\right)$ \\
\hline$f[x \cos (\alpha)+y \sin (\alpha),-x \sin (\alpha)+y \cos (\alpha)]$ & $\mathcal{F}(u \cos (\alpha)+v \sin (\alpha),-u \sin (\alpha)+v \cos (\alpha))$ \\
\hline
\end{tabular}

where the RBC showed their native shapes without applied forces during sedimentation [3]. With only 5062 images available, we do not subdivide the dataset into a single training and test set. Instead, we make use of a cross-validation approach in our experiments, that is we subdivide the data into 10 subsets. We then use each set for testing while the remaining nine sets are used for training, with the overall error rate being the mean over all subset error rates. Note that although we use all images as test and training images, the according training and test sets are strictly disjoint in all cases. A drawback of the RBC database is the lack of results obtained by competing classification methods. Therefore, to prove the efficiency of our classifier, we also apply it to the widely used USPS database (ftp://ftp.mpik-tueb.mpg.de/pub/bs/data/), containing 7291 training and 2007 test samples of isolated, handwritten digits, which are represented by a $16 \times 16$ pixels sized grayscale image. The USPS recognition task is known to be hard, with a human error rate of about $2.5 \%$ on the testing data [4].

\section{Feature Analysis}

In our RBC experiments, we make use of a Fourier-Mellin based feature analysis [5], of which we give a short overview here. The discrete Fourier transformation $(\mathrm{FT}) \mathcal{F}(u, v)$ of a $2 \mathrm{D}$ discrete image $f[x, y] \in \mathbb{R}^{N \times N}$ is defined as

$$
\mathcal{F}(u, v)=\frac{1}{N^{2}} \sum_{j=0}^{N-1} \sum_{k=0}^{N-1} f[j, k] \cdot \exp \left(-\frac{2 \pi i(u j+v k)}{N}\right)
$$

with $i=\sqrt{-1}$ and $u, v=0,1, \ldots, N-1$. Using the FT properties shown in Table 1, the following characteristics of the amplitude spectrum $A$ of $\mathcal{F}(u, v)$ can be derived: it is invariant with respect to translation, inverse-variant with respect to scaling and variant with respect to rotation. Thus, features based on the amplitude spectrum of an image are translation invariant. By transforming

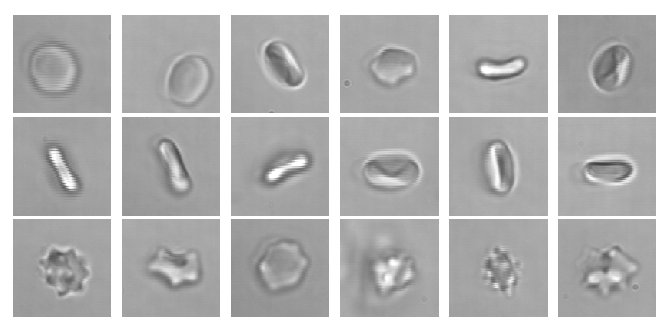

Figure1. RBC example images, top to bottom: stomatocytes, discocytes, echinocytes. 


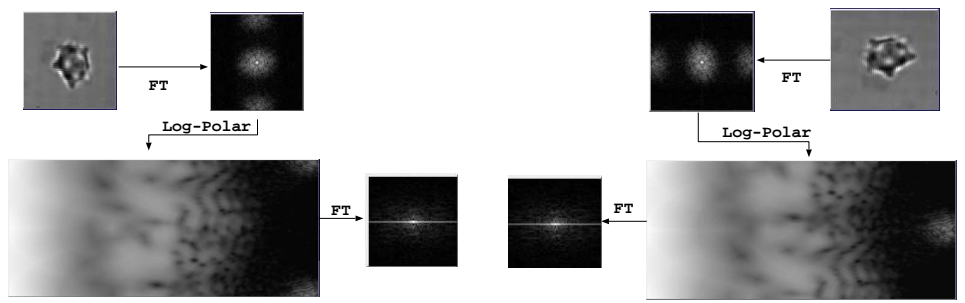

Figure2. RST-invariant feature extraction: A rotation example. Note that the image rotation becomes a vertical shift in the log-polar plane.

the rectangular coordinates $(u, v)$ of $A(u, v)$ to polar coordinates $(r, \theta)$ and by using a logarithmic scale for the radial axis, image scales and rotations become shifts in the $\log$-polar representation $\hat{A}$ of $A$. Now, by computing the amplitude spectrum $B$ of the FT of $\hat{A}$, we can extract features which are RST-invariant (see Figure 2). As $\hat{A}$ is real valued, $B$ is symmetric. Therefore, we extract $24 \times 12$ features from $B$ employing a low-pass filtering under consideration of symmetry, which yields 288-dimensional feature vectors. Thus, by computing these FourierMellin features, classification is not only RST invariant, but we also reduce the dimensionality of the feature space from $128 \times 128=16384$ to 288 . To further decrease the number of model parameters (increasing the reliability of parameter estimation), we use a linear discriminant analysis (LDA) [6, pp.114123 ] for feature reduction. As we can only extract a maximum of $(K-1)$ features using an LDA (with $K$ being the number of classes), we create pseudoclasses by applying a cluster analysis to the training data, where each cluster is considered a pseudoclass. Creating 16 pseudoclasses per class, we extract $(3 \times 16)-1=47$ LDA features. These features are then used to train the model parameters. In the case of USPS, we skip the RST feature analysis (a full rotation invariance is not desired in digit recognition, as we would confuse ' 6 ' and ' 9 ' for instance) and compute 39 LDA features from the original images using 4 pseudoclasses.

\section{Gaussian Mixture Densities in Bayes Context}

To classify an observation $x \in \mathbb{R}^{d}$ we use the Bayesian decision rule [6, pp.10-39]

$$
x \longmapsto r(x)=\underset{k}{\operatorname{argmax}}\{p(k) p(x \mid k)\}
$$

where $p(k)$ is the a priori probability of class $k, p(x \mid k)$ is the class conditional probability for the observation $x$ given class $k$ and $r(x)$ is the classifier's decision. As neither $p(k)$ nor $p(x \mid k)$ are known, we have to choose models for them and estimate their parameters using the training data. In our experiments, we estimate $p(k)$ via relative frequencies and model $p(x \mid k)$ by using GMD, being a linear combination of Gaussian component densities $\mathcal{N}\left(x \mid \mu_{k i}, \Sigma_{k i}\right)$ :

$$
p(x \mid k)=\sum_{i=1}^{I_{k}} c_{k i} \cdot \mathcal{N}\left(x \mid \mu_{k i}, \Sigma_{k i}\right)
$$

where $I_{k}$ is the number of component densities used to model class $k, c_{k i}$ are 
Table2. Results reported on USPS

\begin{tabular}{|l|r|}
\hline Method & Error Rate [\%] \\
\hline \hline Human Performance [4] & 2.5 \\
\hline \hline Two-Layer Neural Net [8] & 5.9 \\
\hline 5-Layer Neural Net (LeNet1) [9] & 4.2 \\
\hline Invariant Support Vectors [10] & 3.0 \\
\hline \hline This work: Gaussian Mixture Densities & 4.5 \\
Gaussian Mixture Densities, VTS & 3.4 \\
\hline
\end{tabular}

weight coefficients (with $c_{k i}>0$ and $\sum_{i} c_{k i}=1$ ), $\mu_{k i}$ is the mean vector and $\Sigma_{k i}$ is the covariance matrix of the component density $i$ of class $k$. To avoid the problems of estimating a covariance matrix in a high-dimensional feature space, i.e. to keep the number of parameters to be estimated small, we make use of globally pooled covariance matrices in our experiments, that is we only estimate a single $\Sigma$, i.e. $\Sigma_{k i}=\Sigma \forall k=1, \ldots, K$ and $\forall i=1, \ldots, I_{k}$. Furthermore, we only use a diagonal covariance matrix, i.e. a variance vector. Note that this does not necessarily imply a loss of information, as a mixture density of that form can still approximate any density function with arbitrary precision. Maximum-likelihood parameter estimation is now done using the Expectation-Maximization (EM) algorithm. More information on this topic can be found in [7].

\section{Results}

We started our RBC experiments by using each image pixel as a feature. Not surprisingly, we were not able to achieve an error rate below $31 \%$ (averaged over all 10 subsets). Extracting 288 RST-invariant features (as described above) without performing an LDA reduced the error rate to $18.8 \%$. This error rate could further be reduced to $15.3 \%$ by using $47 \mathrm{LDA}$ features (with a total of about 200 component densities). Finally, by using a simple reject rule (the likelihood of the 'best' class must be at least $20 \%$ better than that of the second best), the error rate could be reduced to $13.6 \%$ at $2.4 \%$ reject, with the subset error rates ranging from $10.7 \%$ to $16.1 \%$. Note that a single view of an $\mathrm{RBC}$ often provides only poor information for classification (e.g. in some cases, stomatocytes and discocytes are hard to distinguish). Therefore, it seems necessary to classify image sequences rather than single images to further reduce this error rate. We also applied our approach to the USPS task. Using \pm 1 pixel shifts, we created virtual training and testing data. The virtual training data $(65.619$ samples) was then used to train our classifier, whereas the virtual test samples (VTS) were used to come to a combined decision for the original test sample [11]. Using a total of about 8000 component densities, we obtained an excellent error rate of $3.4 \%$ ( $4.5 \%$ without VTS). A comparison of our results with that achieved by other state-of-the-art classifiers can be found in Table 2 .

\section{Conclusion}

In this paper we presented a statistical approach to classifying red blood cells. Having extracted RST-invariant features using a Fourier-Mellin transformation 
based approach, we modelled the observed data using Gaussian mixture densities, where classification was done using the Bayes rule. We obtained an error rate of $13.6 \%$, which is considerably lower than the human error rate of $>20 \%$ (proving the hardness of the problem). We also applied our approach to the US Postal Service handwritten digits recognition task, obtaining an excellent error rate of $3.4 \%$. We are currently working on improving the invariance properties of our classifier by incorporating distance measures which are invariant with respect to affine transformations (such as SimARD's tangent distance [4]). First results on classifying radiographs are very promising.

\section{Acknowledgement}

The authors wish to thank Mrs. H. Horstkott and Mrs. R. Degenhardt, Department of Physiology, RWTH Aachen, for manually classifying the RBC images used in our experiments.

\section{References}

1. B. Deuticke, R. Grebe, C. Haest, "Action of Drugs on the Erythrocyte Membrane", in: Blood Cell Biochemistry, Vol. 1: Erythroid Cells. J.R. Harris, editor, Plenum Press, New York, pp. 475-529, 1990.

2. T. Fischer, Department of Physiology, RWTH Aachen University of Technology, personal communication, 1999.

3. M. Schoenfeld, R. Grebe, "Automatic Shape Quantification of Freely Suspended Red Blood Cells by Isodensity Contour Tracing and Tangent Counting", Computer Methods and Programs in Biomedicine, Vol. 28, Elsevier, pp. 217-224, 1989.

4. P. Simard, Y. Le Cun, J. Denker, "Efficient Pattern Recognition Using a New Transformation Distance," S.J. Hanson, J.D. Cowan, C.L. Giles (eds.): Advances in Neural Information Processing Systems 5, Morgan Kaufmann, San Mateo CA, pp. 50-58, 1993.

5. B. Shrinivasa Reddy, B. Chatterji, "An FFT-based Technique for Translation, Rotation and Scale invariant Image Registration," IEEE Transactions on Image Processing, Vol. 5, August 1996.

6. R. Duda, P. Hart, Pattern Classification and Scene Analysis, Wiley \& Sons, 1973

7. J. Dahmen, K. Beulen, H. Ney, "Objektklassifikation mit Mischverteilungen," 20. DAGM Symposium Mustererkennung 1998, pp. 167-174, Stuttgart, Germany, 1998.

8. V. Vapnik. The nature of Statistical Learning Theory, Springer, New York, pp. 142-143, 1995.

9. P. Simard, Y. Le Cun, J. Denker, B. Victorri. "Transformation Invariance in Pattern Recognition - Tangent Distance and Tangent Propagation," Lecture Notes in Computer Science, Vol. 1524, Springer, pp. 239-274, 1998.

10. B. Schölkopf, P. Simard, A. Smola, V. Vapnik. "Prior Knowledge in Support Vector Kernels," M. Jordan, M. Kearns and S. Solla, editors, Advances in Neural Information Processing Systems 10, MIT Press, pp. 640-646, 1998.

11. J. Dahmen, R. Schlüter, H. Ney. "Discriminative Training of Gaussian Mixtures for Image Object Recognition," 21. DAGM Symposium Mustererkennung 1999, pp. 205-212, Bonn, Germany, 1999. 